\title{
Retinopatia de Purtscher e Purtscher-like
}

\section{Purtscher's and Purtscher-like retinopathies}

Hilton Arcoverde Gonçalves de Medeiros', João Eugenio Gonçalves de Medeiros², Larissa Casteluber Caliari', Jaquison Furtado da Silva ${ }^{4}$

\begin{abstract}
Resumo
A retinopatia de Purtscher é uma condição caracterizada pela baixa súbita de acuidade visual precedida por traumatismo, principalmente torácico, podendo aparecer em outros tipos de traumatismos. Uma entidade com o mesmo quadro clínico descrita como retinopatia "Purtscher-like" apresenta características semelhantes, no entanto na inexistência de um trauma direto. Neste caso pode vir associada a fenômenos tromboembólicos, secundária a pancreatite aguda, pós-manobra de Valsalva, pósanestesia retrobulbar, e outros. O achado oftalmológico comum é a presença de manchas esbranquiçadas no polo posterior com hemorragias peripapilares, associadas à papiledema e áreas de não perfusão capilar. Neste artigo, revisamos os critérios diagnósticos, os padrões nos exames complementares e tratamentos utilizados em cada caso.
\end{abstract}

Descritores: Doenças retinianas/etiologia; Doenças retinianas/diagnóstico; Pancreatite/ complicações; Transtornos da visão; Acuidade visual; Hipertensão intracraniana;Edema macular; Anestesia epidural/efeitos adversos; Ferimentos e lesões/complicações

\footnotetext{
${ }^{1}$ Médico-oftalmologista da Clínica de Olhos Dr. João Eugenio, Doutor em oftalmologia pela UnB - Universidade de Brasília (DF), Brasil; ${ }^{2}$ Pós-Graduado no Instituto Barraquer (Espanha), Fellow em Retina na Retina Service Boston (USA); Médico-oftalmologista da Clínica de Olhos Dr. João Eugenio - Brasília (DF), Brasil;

Residente (R3) de Oftalmologia da Clínica de Olhos Dr. João Eugenio - Brasília (DF), Brasil;

${ }^{4}$ Residente (R2) de Oftalmologia da Clínica de Olhos Dr. João Eugenio - Brasília (DF), Brasil.

Trabalho realizado na Clinica de Olhos Dr. João Eugenio, Brasília (DF), Brasil.
} 


\section{INTRODUÇÃO}

A retinopatia de Purtsher (RP) foi primeiramente descrita por Otmar Purtscher em 1910. No seu trabalho original ${ }^{(1)}$, o autor relata aparecimento de hemorragias intrarretiniana e lesões esbranquiçadas com perda transitória de acuidade visual (AV), secundária a uma queda.

Desde então, diversos autores têm publicado casos semelhantes com variadas etiologias, como traumatismos torácicos, pancreatites, insuficiência renal, embolia gordurosa, anestesia retrobulbar, doenças linfoproliferativas, transplante renal, trauma por "air bag", manobra de Valsava, pós-parto, adenocarcinoma de pâncreas, doença do tecido conectivo, e à prática de bungee jumping ${ }^{(2.7)}$.

O termo "Retinopatia Purtscher-like" foi introduzido por Inkeles ${ }^{(8)} \mathrm{em} 1975$ para descrever quadro clínico idêntico a RP, em um caso de pancreatite aguda, na ausência de traumatismo prévio.

Classicamente a recuperação da acuidade visual, seja ela total ou parcial, é verificada entre 2 e 6 meses após o inicio dos sintomas ${ }^{(8)}$.

Epidemiologicamente, se estima uma prevalência baixa de 0,24 casos por milhão de habitantes ${ }^{(9)}$,e destes, aproximadamente $50 \%$ terá ganho de pelo menos 2 linhas na escala de Snellen nos primeiros 2 meses.

\section{Quadro clínico}

Normalmente a queixa principal de um paciente com RP é baixa de acuidade visual, com intensidade variável, podendo ir desde leve a severa. Esta perda de AV pode se manifestar concomitantemente com o aparecimento da doença sistêmica, ou se manifestar tardiamente, após poucas horas a alguns dias ${ }^{(8)}$.

A oftalmoscopia mostra a papila com perda do contorno, evidenciando uma exsudação papilar ou um papiledema, presença de manchas esbranquiçadas, tipo exsudados algodonosos, associadas a hemorragias intrarretiniana superficiais em "chama de vela", podendo coexistir com hemorragias puntiformes profundas, normalmente contidas dentro de uma zona de 10 diâmetros papilares. As manchas esbranquiçadas aparecem como dispersas e múltiplas, quase sempre localizadas próximo ao território venular, intrarretiniana com contornos bem delimitados e dimensões variadas, podendo-se encontrar um reflexo semelhante ao de "vermelho cereja" macular, caso as manchas acometam o polo posterior e envolvam a macula ${ }^{(10)}$.

O exame angiofluoresceinográfico revela oclusão pré-capilar, com áreas de hipofluorescência por bloqueio da hiperfluorescência coroideana, correspondendo às manchas esbranquiçadas (Figura 1); em alguns casos encontra-se hiperfluorescência tardia nestas áreas. Pode haver pequenos territórios de não perfusão capilar, ao redor do nervo óptico, o qual pode apresentar extravasamento de corante nas fases tardias do angiograma a partir dos capilares do leito acometido.

A circulação coroideana também se encontra comprometida, podendo ser melhor estudada na angiografia com indocianina verde, que apresenta padrão de hipofluorescência no leito coroideano correspondente as manchas esbranquiçadas vistas na retinografia. Tal hipofluorescência persiste por várias semanas, mesmo após a melhora das manchas retinianas, comprovando um envolvimento vascular coroideano importante, apesar das anastomoses presentes na vascularização coroideana ${ }^{(12)}$.

A tomografia de coerência óptica (OCT) revela uma refletividade alterada das camadas retinianas com atenuação da camada nuclear externa, desorganização do complexo epitélio pigmentado retiniano/segmento externo dos fotorreceptores, aumento da espessura retiniana e acúmulo de fluido sub-retiniano, principalmente peripapilar, na fase aguda da alteração. Com exames seriados pode-se acompanhar a resolução completa em algumas semanas ${ }^{(13)}$ (Figura 2).

$\mathrm{Na}$ fase inicial, a eletrofisiologia multifocal evidencia depressão das ondas A e B nas áreas correspondentes as lesões esbranquiçadas, sem, no entanto, haver reduções seletivas da onda B. Após a resolução do quadro inicial, observa-se significante melhora da resposta retiniana com aumento das ondas A e B.

A histopatologia evidencia áreas de oclusão vascular retiniana com edema das capas internas retinianas, espaço cistóide sub-retiniano, com transição súbita para a normalidade ${ }^{(14)}$. Nestas áreas pode haver perda do segmento externo dos elementos fotorreceptores, com epitélio pigmentado retiniano adjacente normal. Pode-se encontrar oclusão ao nível da circulação coroideana, porém sem que esta seja responsável pelo quadro retiniano, uma vez que o epitélio pigmentado se encontra normal.

\section{Fisiopatologia}

O mecanismo fisiopatológico exato ainda é controverso. A hipótese mais aceita é a de um microêmbolo levando a oclusão pré-capilar arteriolar e consequente infarto da microvasculatura na camada de fibras nervosas, com hemorragias nas capas retinianas externas ${ }^{(14,15)}$, embora alguns autores postulem como causa inicial um dano endotelial capilar ${ }^{(111,15)}$. 

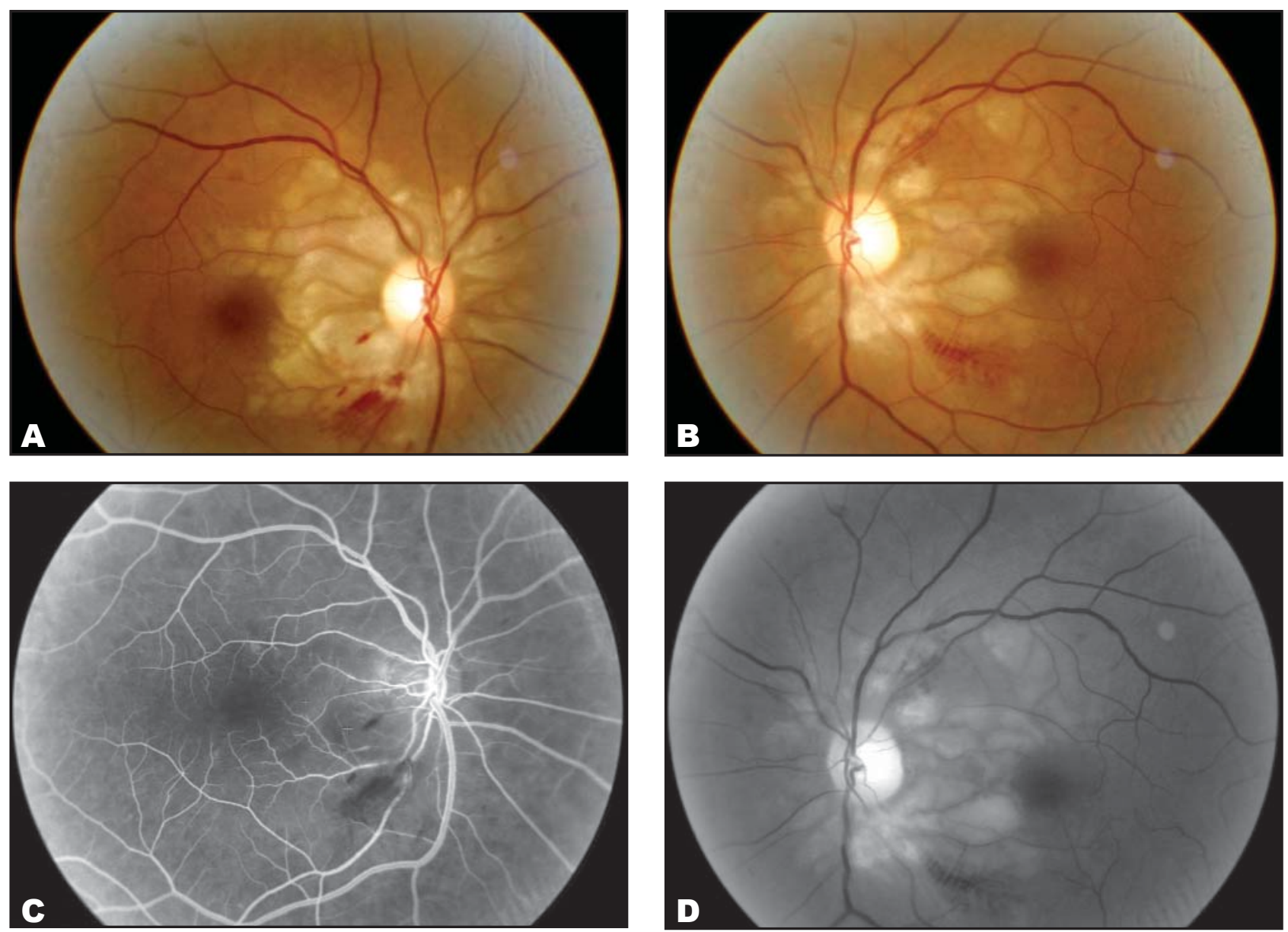

Figura 1: Paciente submetida à anestesia tipo bloqueio peridural. Após o procedimento anestésico, a paciente iniciou alterações neurológicas severas evoluindo para o coma, que persistiu por 24h. Após este período, houve melhora do quadro neurológico, queixando-se de perturbação oftalmológica, com perda acentuada da acuidade visual (20/400 em ambos os olhos). Figuras A e B: Retinografia revela polo posterior esbranquiçado devido à presença de manchas algodonosas confluentes e pequenas hemorragias peripapilares em AO; Figura C: A angiografia fluoresceínica mostra oclusão pré-capilar peripapilar; Figura D: Aspecto autofluorescente das lesões esbranquiçadas, vistas à luz aneritra

A origem desse êmbolo é contraditória e pode variar de acordo com a patologia. Poderia ser embolia gordurosa, ou relacionada à liberação, na circulação sistêmica, de enzimas proteolíticas procedentes do pâncreas inflamado ${ }^{(16,17)}$.

Tem-se proposto que a agregação leucocitária induziria a ativação da cascata do complemento. Nestes casos, as manchas esbranquiçadas retinianas podem ser atribuídas a um extravasamento linfático secundário a um aumento da agregação de granulócitos, como resposta a ativação da fração 5 do complemento ${ }^{(18)}$. Trabalhos experimentais demonstraram a capacidade de agregação granulocitária do fator 5 do complemento uma vez que $\mathrm{o}$ anticorpo anti-C5 inibe completamente a agregação ${ }^{(19)}$.

Esta hipótese é bastante aceita por explicar tanto as lesões de origem traumática, quanto em processos inflamatórios e doenças vasculares. Outros êmbolos potenciais associados à RP seriam de ar, plaquetas, de fibrina ou de fluido amniótico. Na síndrome associada ao pós-parto, a RP estaria relacionada com a provável embolização do fluido amniótico ou com a coagulação intravascular induzida por leucoêmbolos secundários a ativação do complemento ${ }^{(20)}$.

As alterações retinianas associadas a práticas esportivas do bungee jumping ${ }^{(21)}$ apresentam características semelhantes a RP, sendo causadas pelo brusco aumento da pressão intravascular nas porções superiores do corpo devido a gravidade e a súbita desaceleração que ocorre no final da queda. Como consequência ocorre o aumento súbito da pressão intratorácica causando elevação repentina da pressão venosa nos olhos e ruptura dos capilares retinianos originando micro hemorragias ou descolamento hemorrágico da membrana limitante 

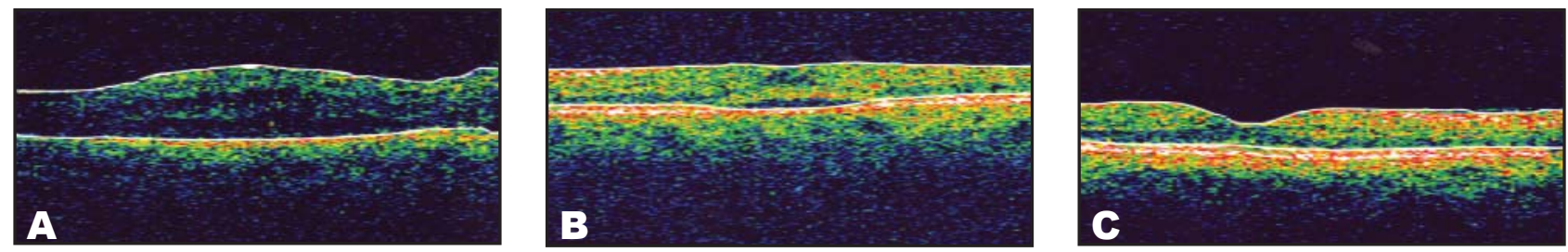

Figura 2: A: tomografia de coerência óptica (Zeiss Stratus OCT Ver 3.0, Carl Zeiss Meditec, Dublin, CA) inicial revelando edema macular bilateral, com acúmulo de fluido sub-retiniano, principalmente na região peripapilar; B: 30 dias após, melhora do quadro anterior com reabsorção parcial do fluido sub-retiniano, persistindo pequeno fluido na região macular; C: Exame com 1 ano, evidenciando resolução completa do fluido sub-retiniano e aspecto normal da região macular

interna na região foveolar ${ }^{(22,23)}$.

Pode haver, ainda, uma forma de oclusão sem embolização, por aumento súbito da pressão intracraniana, com refluxo liquórico pelo nervo óptico e lesão endotelial hipertensiva, produzindo aumento da pressão no nível da lamina cribosa, levando a oclusão pré-capilar ${ }^{(1724)}$, o que justificaria quadros como nas manobras de Valsava, nos pós-anestesias retrobulbares e pós-acidentes anestésicos peridurais.

Estes casos podem ser melhor compreendidos analisando-se os quadros de RP relacionados com anestesia peridural. Esta se diferencia da anestesia raquidiana principalmente por: 1) a quantidade e o tipo de anestésico envolvido serem diferentes, sendo um volume muito maior na anestesia peridural que na raquidiana; 2) não penetrar no espaço subaracnóideo; 3) possuir tempo de latência maior, não estando indicada nos procedimentos em que se necessita de rapidez anestésica ${ }^{(25)}$. Durante uma anestesia peridural, a perfuração acidental, não diagnosticada, da membrana aracnóide, com consequente injeção de um grande volume de anestésico no espaço liquórico, produz um súbito aumento da pressão intracraniana, podendo desencadear um quadro retiniano de retinopatia "Purtscher-like", compatível com o postulado por Marr ${ }^{(24)}$ e Duane ${ }^{(26)}$.

De todas as formas, o mecanismo final comum é a alteração da permeabilidade microvascular retiniana secundária a uma micro-oclusão capilar, com lesão endotelial hipertensiva ${ }^{(16)}$.

\section{Diagnóstico diferencial}

O diagnóstico RP e RPL deve ser lembrado sempre que o paciente apresentar histórico de trauma recente, principalmente torácico, ou na existência de alterações sistêmicas capazes de produzir fenômenos tromboembólicos, aumento súbito da pressão intracraniana, ou, aumento da pressão intraorbitária.

De todas as patologias que podem entrar no diagnóstico diferencial, a oclusão da artéria central, e da ar- téria ciliorretiniana são as mais importantes pois demandam cuidados imediatos.

Em alguns casos, a oclusão da artéria cilioretiniana pode apresentar quadro retiniano extremamente parecido a uma retinopatia de Purstcher, porém não haverá hemorragias superficiais, nem papiledema.

A artéria ciliorretiniana (ACR) se origina das curtas ciliares posteriores e, raramente, dos vasos $\operatorname{coróideos}^{(27)}$. Nutre a retina interna peripapilar, mormente a região interpapilamacular. Classicamente, apresenta na angiografia fluoresceínica um enchimento simultâneo com a circulação coroideana, precedendo o enchimento da artéria central da retina ${ }^{(27,28)}$.

A oclusão da ACR pode ocorrer isoladamente em $5 \%$ dos casos de obstruções arteriolares retinianas e geralmente relacionada com ateromatose carotídea, ou associada à oclusão da veia central da retina, com prognóstico visual ruim, provavelmente pela atrofia óptica que se desenvolve ${ }^{(29,30)}$. Recentemente, tem sido descrita a associação combinada de oclusão da ACR e NOIA, relacionada com overdose de sildefanil citrato ${ }^{(31)}$ (Viagra Pfizer Pharmaceuticals, New York, NY) e em casos de displasia fibromuscular da artéria renal com hipertensão arterial secundária ${ }^{(22)}$. Também há citação de oclusão combinada de ACR e NOIA em pacientes do sexo feminino em uso de reposição hormonal ${ }^{(33)}$.

No exame de fundo de olho, se observa na oclusão da ACR, edema retiniano no pólo posterior, com retina de aspecto isquêmico, na área correspondente à irrigação da ACR, podendo ser encontrado o aspecto fundoscópico "vermelho cereja" na região macular. $\mathrm{Na}$ angiofluoresceinografia, há retardo no preenchimento da ACR e presença de áreas de não perfusão capilar correspondendo à sua vascularização.

\section{Tratamento e evolução}

Em geral o prognóstico é bom, com boa recuperação visual, porém quando há acometimento da circulação venosa papilar, o nervo óptico tende a evoluir para atrofia $^{(10)}$ (Figura 3), ainda que parcial. Outra possibili- 

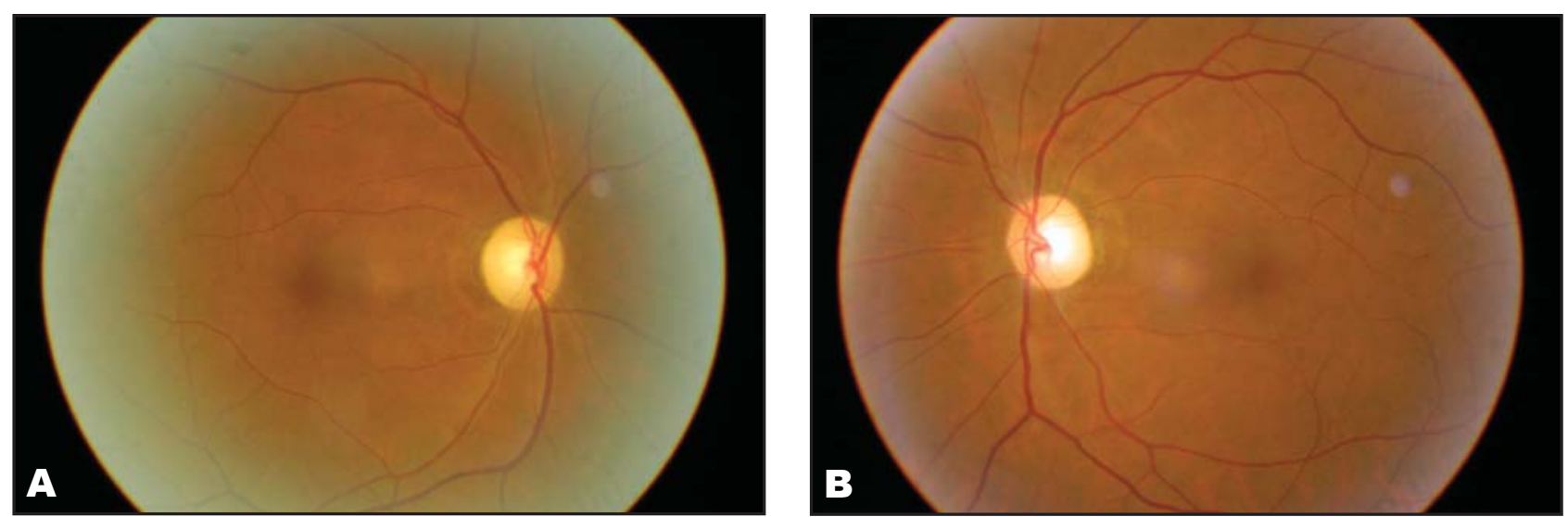

Figura 3: A e B - Retinografia de controle com 1 ano após a lesão, olho D e olho E respectivamente; A AV se encontrava em 20/25 em OD e 20/30 em OE; Observar persistência de palidez papilar

dade de diminuição permanente da acuidade visual ocorre quando a obstrução vascular envolve os capilares da arcada foveolar macular.

O tratamento da retinopatia de Purtscher é realizado com a melhora das condições sistêmicas do paciente. $\mathrm{O}$ uso de corticóides sistêmicos para diminuir a resposta inflamatória é empírico e controverso ${ }^{(34)}$, estando indicado quando fundamental para melhora nas condições clínicas do paciente, ou quando ocorre retardo na resolução do quadro oftalmológico.

$\mathrm{O}$ uso de outras drogas, como vasodilatadores e antiagregante plaquetário, não está indicado, salvo quando necessário para o tratamento da doença de base.

\section{ResUmo}

Purtscher's retinopathy is a condition characterized by loss of vision within hours after an injury, especially thoracic. The term "Purtscher-like retinopathy" is used to describe a similar retinal appearance in a variety of conditions other than trauma. This condition have been reported in acute pancreatitis, lymphoproliferative disorders, chest compression, fat embolization, Valsalva maneuver, after retrobulbar anesthesia, and others. Fundus abnormalities include cotton-wool spots, retinal hemorrhage, non-capillary perfusion areas, and optic disk swelling. In this article we revise the ophthalmoscopic findings, the pattern of complimentary examinations and treatment indicated in each case.

Keywords: Retinal diseases/etiology; Retinal diseases/diagnosis; Pancreatitis/complications; Vision disorders; Visual acuity; Intracranial hypertension/complicações; Macular edema; Anesthesia, epidural/adverse effects; Wounds and injuries/complications

\section{REFERÊNCIAS}

1. Purtscher O. Noch unbekannte Befunde nach Schadeltrauma. Berl Dtsch Ophthalmol Ges. 1910;36:294-301.

2. Schenk C, Holak H, Holak N, Olinger A. [Purtscher's syndrome. An example of a rare occurrence of ocular complication after thoracic compression trauma]. Unfallchirurg. 2005;108(2):1679. German.

3. Tabandeh H, Rosenfeld PJ, Alexandrakis G, Kronish JP, Chandhry NA. Purtscher-like retinopathy associated with pancreatic adenocarcinoma. Am J Ophthalmol. 1999;128(5):650-2.

4. D’Alessandro L, Mejía R, Malbrán E, Karol E. [Retinopathy associated with acute pancreatitis]. Medicina (B Aires). 1994;54(4):340-2.

5. Lim BA, Ang CL. Purtscher-like retinopathy after retrobulbar injection. Ophthalmic Surg Lasers. 2001;32(6):477-8

6. Diniz JR, Arantes TEF, Urbano RV, Danda DMR, Almeida DD. Alterações oculares associadas ao "bungee jumping" relato de caso. Arq Bras Oftalmol. 2005;68(6):853-6.

7. Okwuosa TM, Lee EW, Starosta M, Chohan S, Volkov S, Flicker $\mathrm{M}$, et al. Purtscher-like retinopathy in a patient with adult-onset Still's disease and concurrent thrombotic thrombocytopenic purpura. Arthritis Rheum. 2007;57(1):182-5.

8. Inkeles DM, Walsh JB. Retinal fat emboli as sequela to acute pancreatitis. Am J Ophthalmol. 1975;80(5):935-8.

9. Holló G. Frequency of Purtscher's retinopathy. Br J Ophthalmol. 2008;92(8):1159. Comment on: Br J Ophthalmol. 2007;91(11):1456-9.

10. Agrawal A, McKibbin M. Purtscher's retinopathy: epidemiology, clinical features and outcome. Br J Ophthalmol. 2007;91(11):1456-9. Comment in: Br J Ophthalmol. 2008;92(8):1159.

11. Gass JDM. Atlas of macular disease. Diagnosis and treatment. 2nd ed. St Louis: Mosby; 1977. p. 1096-105.

12. Gomez-Ulla F, Fente B, Torreiro M, Salorio MS, Gonzalez F. Choroidal vascular abnormality in Purtscher's retinopathy shown by indocyanine green angiography. Am J Ophthalmol.1996;122(2):261-3.

13. Soliman W, Zibrandtsen N, Jørgensen T, Sander B, Alsbirk PH, Larsen M. Sequels of Purtscher's retinopathy imaged by enhanced optical coherence tomography. Acta Ophthalmol Scand. 2007;85(4):450-3. 
14. Kincaid MC, Green WR, Knox DL, Mohler C. A clinicopathological case report of retinopathy of pancreatitis. Br J Ophthalmol. 1992;66(4):219-26.

15. Stewart MW, Brazis PW, Guier CP, Thota SH, Wilson SD. Purtscher-like retinopathy in a patient will HELLP syndrome. Am J Ophthalmol. 2007;143(5):886-7

16. Holak H, Holak N, Huzarska M, Holak S. [Pathogenesis of Purtscher's retinopathy and Purtscher-like retinopathy]. Klin Oczna. 2007;109(1-3):38-45. Polish.

17. Carrera CRL, Pierre LM, Medina FMC, Pierre-Filho PTP. Purtscher-like retinopathy associated with acute pancreatitis. São Paulo Med J. 2005;123(6): 289-91.

18. Sellami D, Ben Zina Z, Jelliti B, Abid D, Feki J, Chaâboun M. Pseudorétinopathie de Purtscher révélant un lupus érythémateux dissemine. J Fr Ophtalmol. 2002;25(1):52-5.

19. Craddock PR, Hammerschmidt D, White JG, Dalmasso AP, Jacob HS. Complement (C5-a)-induced granulocyte aggregation in vitro. A possible mechanism of complementmediated leukostasis and leukopenia. J Clin Invest. 1977;60(1):260-4.

20. Blodi BA, Johnson MW, Gass JD, Fine SL, Joffe LM. Purtscher's-like retinopathy after childbirth. Ophthalmology. 1990;97(12):1654-9

21. Gomes AMV. Manifestações vítreo-retinianas no trauma ocular contuso. In: Lavinsky J, organizador. Doenças prevalentes da retina e vítreo. Rio de Janeiro. Cultura Médica; 2002. p. 302-5.

22. Capão Filipe JA, Rocha-Sousa A, Falcão Reis F, Castro Correia J. Modern sports eye injuries. Br J Ophthalmol. 2003;87(11):1336-9

23. Hite PR, Greene KA, Levy DI, Jackimczyk K. Injuries resulting from bungee-cord jumping. Ann Emerg Med. 1993;22(6):1060-3

24. Marr WG, Marr EG. Some observations on Purtscher's disease: traumatic retinal angiopathy. Am J Ophthalmol. 1962;54:693

25. Aveline C, Bonnet F. [The effects of peridural anesthesia on duration of labor and mode of delivery]. Ann Fr Anesth Reanim. 2001;20(5):471-84. French.
26. Duane TD. Valsalva hemorrhagic retinopathy. Am J Ophthalmol. 1973;75(4):637-42.

27. Hayreh SS. The cilio-retinal arteries. Br J Ophathalmol. 1963;47:71-89.

28. Justice J Jr, Lehmann RP. Cilioretinal arteries. A study based on review of stereo fundus photographs and fluorescein angiographic findings. Arch Ophthalmol. 1976;94(8):1355-8.

29. Brown GC, Shields JA. Cilioretinal arteries and retinal arteries occlusion. Arch Ophthalmol. 1979;97(1):84-92.

30. Brown GC, Moffat K, Cruess A, Magargal LE, Goldberg RE. Cilioretinal artery obstruction. Retina. 1983;3(3):182-7.

31. Akash R, Hrishikesh D, Amith P, Sabah S. Case report: association of combined nonarteritic anterior ischemic optic neuropathy (NAION) and obstruction of cilioretinal artery with overdose of Viagra. J Ocul Pharmacol Ther. 2005;21(4):315-7.

32. Warrasak S, Tapaneya-Olarn W, Euswas A, Sriphojanart S, Sirikulchayanonta V, Leelachaikul P. Fibromuscular dysplasia: a rare cause of cilioretinal artery occlusion in childhood. Ophthalmology. 2000;107(4):737-41.

33. Murray DC, Christopoulou D, Hero M. Combined central retinal vein occlusion and cilioretinal artery occlusion in a patient on hormone replacement therapy. $\mathrm{Br} \mathrm{J}$ Ophthalmol. 2000;84(5):549-50.

34. Atabay C, Kausu T, Nurlu G. Late visual recovery after intravenous methylprednisolone treatment of Purtscher's retinopathy. Ann Ophthalmol. 1993;25(9):330-3.

\section{ENDEREÇO PARA CORRESPONDÊNCIA: \\ Hilton Arcoverde Gonçalves de Medeiros \\ SHIS QI 05 Conjunto 09 - casa 02 \\ Lago Sul - Brasília - DF \\ Fax: (61) 3364-4101 \\ Email: h.medeiros@brturbo.com.br}

